\title{
The Mu2e Experiment at Fermilab
}

\author{
Markus Röhrken*† \\ California Institute of Technology \\ E-mail: roehrken@caltech.edu
}

The Mu2e experiment will search for the coherent neutrino-less conversion of muons to electrons in the field of an atomic nucleus. The sensitivity of Mu2e for this charged lepton flavor violating process will be four orders of magnitudes better compared to previous experiments. The search for charged lepton flavor violation probes for physics beyond the Standard Model on a high effective mass scale, that is not accessible to present or future collider experiments operated at the highest energies. This article presents the physics of Mu2e including prospects on the sensitivity to new physics effects and the experimental setup.

The European Physical Society Conference on High Energy Physics 22-29 July 2015

Vienna, Austria

\footnotetext{
* Speaker.

† On behalf of the Mu2e Collaboration.
} 


\section{Introduction}

Charged lepton flavor violation (CLFV) is extremely suppressed in the Standard Model due to terms summing over $\Delta m_{i j}^{2} / M_{W}^{2}$, where $\Delta m$ is the neutrino mass difference and $M_{W}$ is the mass of the $\mathrm{W}$ boson. For example, the Standard Model constraint on the branching fraction of $\mu \rightarrow e \gamma$ is

$$
\mathscr{B}(\mu \rightarrow e \gamma)=\frac{3 \alpha}{32 \pi}\left|\sum_{i=2,3} U_{\mu i}^{*} U_{e i} \frac{\Delta m_{1 i}^{2}}{M_{W}^{2}}\right|^{2} \leq 10^{-54} .
$$

In the Standard Model, the rates of CLFV processes are below any present or future experimental sensitivity. Many extensions of the Standard Model predict sizable enhanced CLFV rates. These include models of extra dimensions, GUTs, heavy neutrinos, lepto-quarks, little Higgs or supersymmetry [1]. Any detection of a CLFV signal would be an unambiguous evidence for physics beyond the Standard Model.

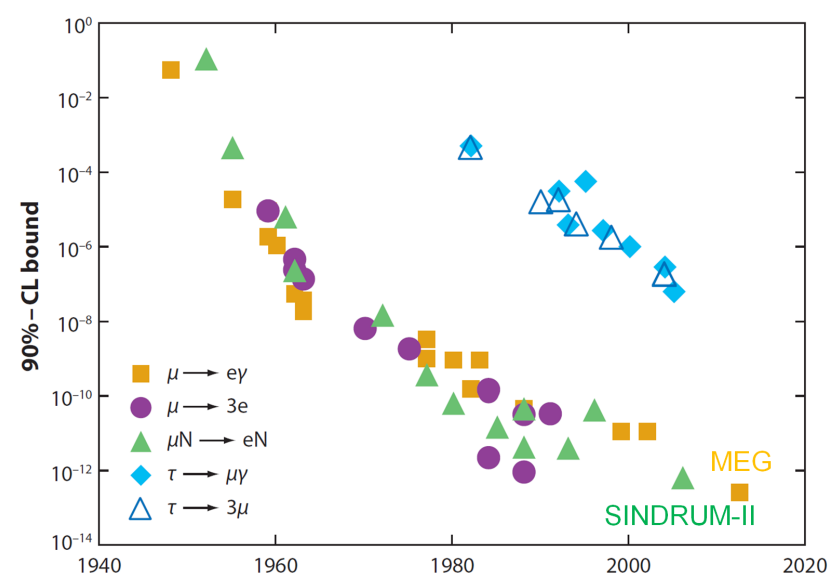

Figure 1: History of searches for CLFV in muon and tau decays [2].

The search for CLFV has a long history as illustrated for muon and tau decays in Figure 1. After pioneering measurements using muons from cosmic rays carried out by Pontecorvo et al. in the late 1940s [3], the experimental sensitivity for CLFV has increased by many orders of magnitude over time. Currently, the most stringent limits come for $\mu \rightarrow e \gamma$ from the MEG experiment $\left(\mathscr{B}(\mu \rightarrow e \gamma)<5.7 \times 10^{-13}\right)[4]$ and for $\mu \rightarrow e N$ conversions from the SINDRUM II experiment $\left(\mathrm{R}_{\mu e}(\mu N \rightarrow e N\right.$ on $\left.\mathrm{Au})<6 \times 10^{-13}\right)[5]$.

The Mu2e experiment being constructed at Fermilab will search for the coherent neutrino-less conversion of muons to electrons in the field of an aluminum nucleus. The conversion process of a muon bound in the 1s state of an aluminum nucleus is illustrated in Figure 2. The $\mu \rightarrow e$ conversion is a coherent process with the kinematics of a two-body decay and has a clear experimental signature. After correcting for the binding energy in the muonic aluminum atom and for the nuclear recoil, the signature is a single mono-energetic $105 \mathrm{MeV}$ electron. Mu2e will measure the rate of 


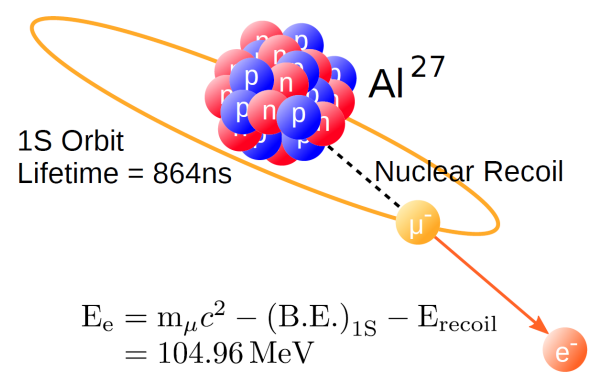

Figure 2: Illustration of the neutrino-less $\mu \rightarrow e$ conversion of a muon bound in the 1s state of an aluminum nucleus.

the $\mu \rightarrow e$ conversion relative to the ordinary muon capture by the nucleus defined as

$$
\mathrm{R}_{\mu e}=\frac{\Gamma\left(\mu^{-}+A(Z, N) \rightarrow e^{-}+A(Z, N)\right)}{\Gamma\left(\mu^{-}+A(Z, N) \rightarrow v_{\mu}+A(Z-1, N)\right)} .
$$

The Mu2e experiment is designed to have a discovery sensitivity of five standard deviations or better for all $\mu \rightarrow e$ conversion rates greater than $2 \times 10^{-16}$. If no signal is observed by Mu2e, the upper limit on the conversion rate is expected to be $6 \times 10^{-17}$ or less at the $90 \%$ confidence level. This is an improvement in sensitivity of four orders of magnitude compared to SINDRUM II.

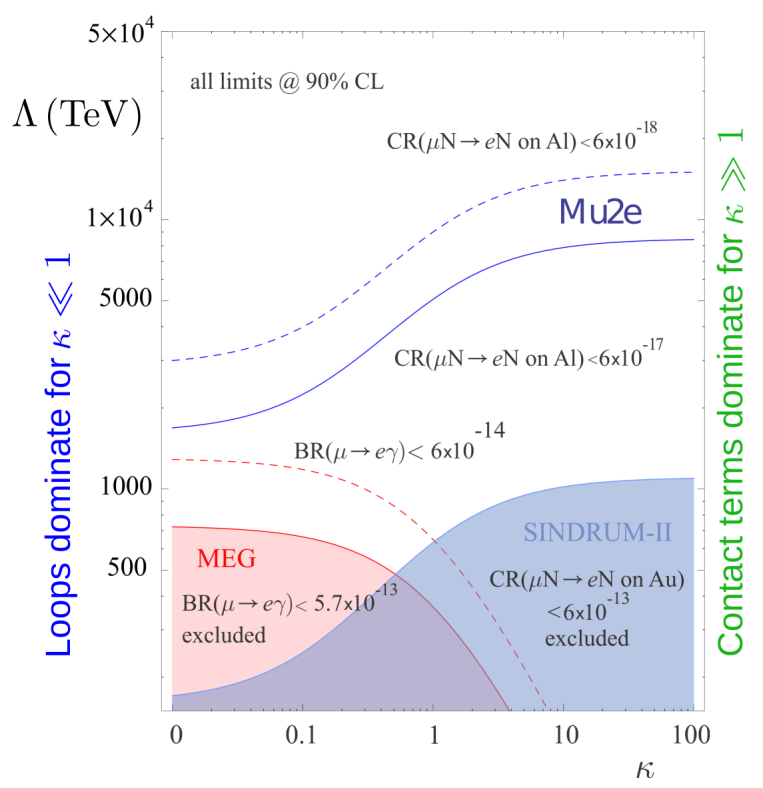

Figure 3: Sensitivity of CLFV processes to physics beyond the Standard Model [6].

The sensitivity of CLFV to physics beyond the Standard Model can be expressed by the modelindependent effective Lagrangian [7]

$$
\mathscr{L}_{\mathrm{CLFV}}=\frac{\mathrm{m}_{\mu}}{(1+\kappa) \Lambda^{2}} \bar{\mu}_{\mathrm{R}} \sigma_{\mu v} \mathrm{e}_{\mathrm{L}} \mathrm{F}^{\mu v}+\frac{\kappa}{(1+\kappa) \Lambda^{2}} \bar{\mu}_{\mathrm{L}} \gamma_{\mu} \mathrm{e}_{\mathrm{L}}\left(\sum_{\mathrm{q}=\mathrm{u}, \mathrm{d}} \overline{\mathrm{q}}_{\mathrm{L}} \gamma^{\mu} \mathrm{q}_{\mathrm{L}}\right)
$$


where $\Lambda$ is the effective mass scale of potential physics beyond the Standard Model and $\kappa$ is a dimensionless parameter, that determines the relative contributions of the two terms. For $\kappa \ll 1$, the first term consisting of a flavor-changing dipole operator dominates. This term gives rise to loop diagrams involving photons, that can become on-shell and contribute to $\mu \rightarrow e \gamma$. For $\kappa \gg 1$, the second term consisting of a four fermion operator dominates. This term gives rise to contact interactions and other processes, that do not result in on-shell photons. The sensitivity to new physics effects for the $\mu \rightarrow e \gamma$ and $\mu \rightarrow e$ processes is illustrated in Figure 3. While the sensitivity for $\mu \rightarrow e \gamma$ is restricted to small values of $\kappa$, the $\mu \rightarrow e$ conversion can probe for new physics effects for a large range of the parameter space of $\kappa$ with best sensitivities for large $\kappa$. Therefore the $\mu \rightarrow e \gamma$ and $\mu \rightarrow e$ processes have complementary sensitivity to new physics effects, and it is important to search for CLFV using both processes. For large $\kappa$, Mu2e will probe effective mass scales up to $10^{4} \mathrm{TeV}$, which is beyond the accessible energies of present or future collider experiments, that directly search for new physics effects.

\section{The Mu2e Experiment}

An overview of the Mu2e experiment is shown in Figure 4. The main components of the Mu2e experiment are three large superconducting solenoids connected in series: First, the production solenoid houses the primary production target made out of tungsten. The production solenoid captures and focuses secondary particles produced by interactions of the incident proton beam with the tungsten target. Second, the transport solenoid transfers low energetic muons through a s-shaped volume, that contains collimators for the momentum and charge selection of charged particles. Third, the detector solenoid contains the aluminum muon stopping target, and detectors for tracking and calorimetry to measure the momenta and energies of charged particles. The solenoids are evacuated to operate the experiment in vacuum. The magnetic field is graded over large parts of the experimental volume ranging from $4.6 \mathrm{~T}$ upstream in the production solenoid to $1 \mathrm{~T}$ downstream, where the tracker and calorimeter are placed.

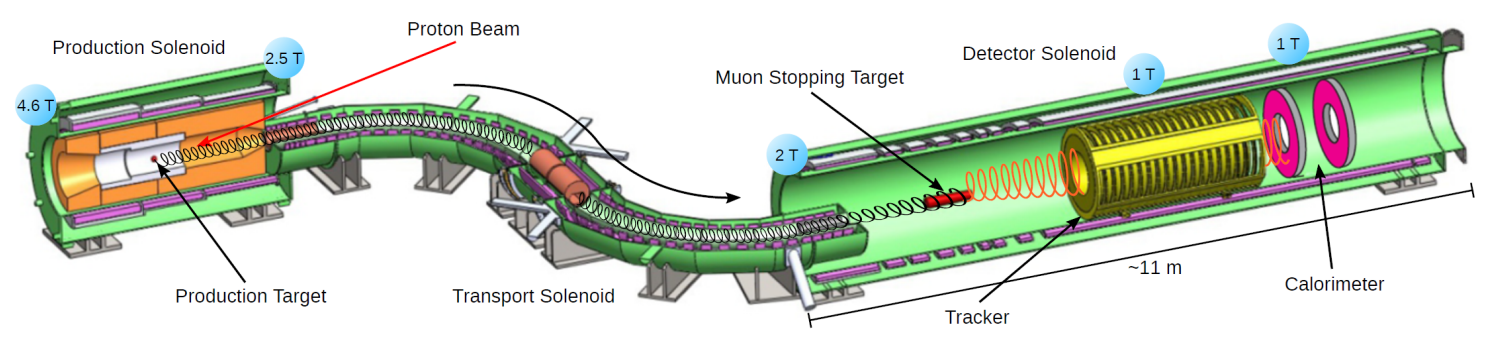

Figure 4: The Mu2e experiment in a cut away view, that highlights the internal components.

A muon stopping target made out of aluminum is placed in the detector solenoid to slow down and stop muons from the low momentum muon beam delivered by the transport solenoid. The stopped muons then form muonic atoms by interactions with the aluminum nuclei of the target. The stopping target is positioned in center of the graded magnetic field of the detector solenoid, which ranges from $2 \mathrm{~T}$ upstream at the entrance of the detector solenoid to $1 \mathrm{~T}$ downstream at the entrance of the tracker. The graded magnetic field acts as a magnetic bottle on the electrons 
emerging from the decays of muons stopped in the target. Electrons leaving the stopping target upstream are deflected by the magnetic bottle and reverse their direction of flight downstream to the tracker.

The tracker is constructed from a low effective mass array of about 20000 straw drift tubes arranged in 18 tracking stations. Each straw tube is $5 \mathrm{~mm}$ in diameter and contains a $25 \mu \mathrm{m}$ sense wire. The straw tubes have $15 \mu \mathrm{m}$ thick walls made out of Mylar and are filled with an Argon- $\mathrm{CO}_{2}$ gas mixture of ratio 80 to 20 . The tracker is $3 \mathrm{~m}$ long and placed inside a uniform magnetic field of $1 \mathrm{~T}$. The tracker measures the momenta of charged particles from the trajectories reconstructed from the hits detected in the straw tubes. The tracker contains a cylindrical inner hole oriented along the beam axis to be insensitive to charged particles with momenta less than $55 \mathrm{MeV} / c$, that originate from the muon beam or from electrons created in Michel decays of muons stopped in the target.

An electromagnetic calorimeter is placed downstream after the tracker. The electromagnetic calorimeter is made out of two disks of scintillating crystals. Each disk contains about $900 \mathrm{BaF}_{2}$ crystals each read about by two avalanche photo-diodes. Similar to the tracker, the electromagnetic calorimeter contains a cylindrical inner hole to be insensitive to low momentum particles from the muon beam or from Michel decays of muons in the stopping target. The calorimeter has a timing resolution of about $500 \mathrm{ps}$ and an energy resolution of about $5 \%$ for $105 \mathrm{MeV}$ electrons, respectively. The electromagnetic calorimeter can independently confirm the momentum measurements by the tracker, contributes to the identification of charged particles and provides fast timing signals for the trigger.

Further instrumentation of the Mu2e experiment is provided by a cosmic ray veto, a monitor to measure the extinction of the proton beam, and a Germanium detector monitoring the stopping target to determine the number of stopped muons captured by the aluminum target, which is important for the normalization of the conversion rate $\mathrm{R}_{\mu e}$. Detailed information on the instrumentation of the Mu2e experiment is provided by Reference [8].
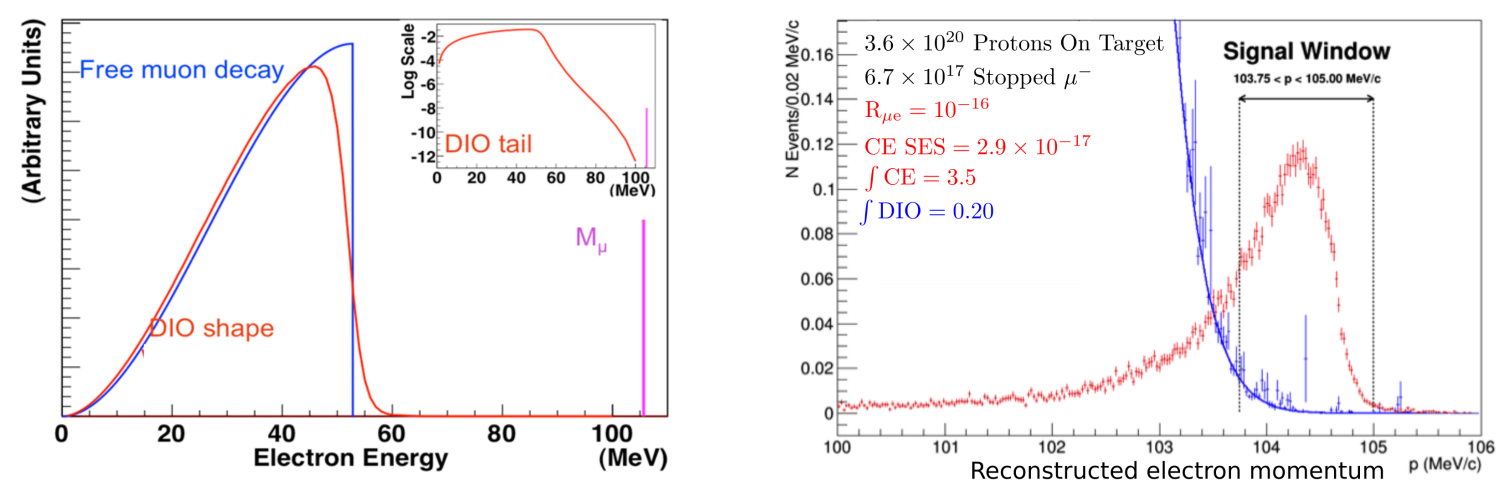

Figure 5: (Left) Momentum spectra for electrons originating from the decay of free muons (blue) and from muons decaying in orbit [DIO] (red). Note the tail for DIO electrons on the high end side of the spectrum close to the endpoint energy (inset). (Right) Reconstructed momentum spectra for electrons from DIOs (blue) and conversion electrons (red) obtained from Mu2e simulations performed for the design luminosity of $3.6 \times 10^{20}$ protons on target and $6.7 \times 10^{17}$ stopped muons, and assuming $\mathrm{R}_{\mu e}=10^{-16}$. 


\section{Detection of the Conversion Signal and Backgrounds}

The essential physics of the Mu2e experiment, that can give rise to the $\mu \rightarrow e$ conversion or to the dominating background processes, takes place in the stopping target. Muons that are stopped in the aluminum target form muonic atoms and quickly cascade down to the 1s state under X-ray emission. In muonic aluminum atoms, the lifetime of the 1s state is $864 \mathrm{~ns}$. About $60 \%$ of the bound muons are captured by the nucleus and about $40 \%$ of the bound muons decay in orbit (DIO) to electrons. The energy spectrum of DIO electrons is similar to that of the Michel decay of free muons with most electrons emitted below $53 \mathrm{MeV}$ as illustrated in Figure 5 (left). However due to recoil effects with the nucleus, the DIO electron spectrum has a tail towards high energies extending the spectrum up to the kinematic endpoint. The DIO electron spectrum falls rapidly for energies close the endpoint, approximately as $\left(\mathrm{E}_{\text {endpoint }}-\mathrm{E}_{e}\right)^{5}$ [9]. The electrons originating from muons bound in the stopping target and decaying in orbit provide the main irreducible background of the Mu2e experiment. In the signal window defined with respect to the momenta of reconstructed conversion electrons, the DIO electrons are expected to contribute 0.20 background events assuming the design luminosity of $3.6 \times 10^{20}$ protons on target and $6.7 \times 10^{17}$ stopped muons. Further background sources are small compared to the DIO background and originate from radiative pion captures, from processes that are induced by cosmic rays or antiprotons, or from muons and pions decaying in flight.

Figure 5 (right) shows the reconstructed electron momenta for DIO and conversion electrons from simulations generated according to the design luminosity of Mu2e corresponding to a physics run of 3 years and assuming a conversion rate of $\mathrm{R}_{\mu e}=10^{-16}$. The experimental signature for the $\mu \rightarrow e$ conversion signal is a peak above the background close to the conversion energy. The reconstructed momentum distribution of $\mu \rightarrow e$ conversion electrons is slightly shifted below the theoretical conversion energy of $105 \mathrm{MeV}$ and has a tail towards the low-energy side, which is caused by multiple Coulomb scattering of the electrons with material of the stopping target and the inner proton absorber, which is placed before the entrance of the tracker. To be able to detect the $\mu \rightarrow e$ conversion signal and to separate it from the background, i.e. from the end of the falling edge of the DIO electron spectrum close to the endpoint, an excellent momentum resolution of the tracker better than a few hundred $\mathrm{keV} / c$ is required.

\section{Summary}

The Mu2e experiment is currently in intensive preparation, and the construction at Fermilab is proceeding on schedule. The commissioning of the experiment is expected for the year 2020 . Mu2e will probe for CLFV with a discovery sensitivity of five standard deviations or better for any $\mu \rightarrow e$ conversion rate larger than $2 \times 10^{-16}$. If Mu2e does not observe a signal, the expected upper limit on the conversion rate is $6 \times 10^{-17}$ or less at the $90 \%$ confidence level. This sensitivity is an improvement of four orders of magnitude compared to previous experiments and promises to discover or to rule out new physics effects for a large variety of models of physics beyond the Standard Model. 


\section{References}

[1] S. Mihara, J.P. Miller, P. Paradisi, G. Piredda, Annu. Rev. Nucl. Part. Sci. 63, 531 (2013).

[2] W.J. Marciano, T. Mori, J.M. Roney, Annu. Rev. Nucl. Part. Sci. 58, 315 (2008).

[3] E.P. Hincks, B. Pontecorvo, Phys. Rev. 73, 257 (1948).

[4] J. Adam et al. (MEG Collaboration), Phys. Rev. Lett. 110, 201801 (2013).

[5] W. Bertl et al. (SINDRUM-II Collaboration), Eur. Phys. J. C 47, 337 (2006).

[6] A. de Gouvêa, Nucl. Phys. B (Proc. Suppl.) 188, 303 (2009).

[7] A. de Gouvêa, P. Vogel, Prog. Part. Nucl. Phys. 71, 75 (2013).

[8] L. Bartoszek et al. (Mu2e Collaboration), "Mu2e Technical Design Report”, Fermilab-TM-2594, arXiv:1501.05241 (2015).

[9] A. Czarnecki, X. Garcia i Tormo, W. Marciano, Phys. Rev. D 84, 013006 (2011). 